\title{
Redes Generativas Adversariais para Detecção de Câncer de Pele
}

\section{William R. Massuda*, Sandra Avila.}

\section{Resumo}

Esse projeto de pesquisa teve como objetivo o estudo de arquiteturas de redes generativas adversariais (GANs) para a geração de imagens sintéticas de lesões de câncer de pele, com foco principal na validação das diferentes métricas de avaliação para essas arquiteturas.

\section{Palavras-chave:}

Redes Generativas Adversariais, Aprendizado Profundo, Melanoma.

\section{Introdução}

Câncer de pele é o tipo de câncer mais comum no mundo. Entretanto, a taxa de sobrevivência cresce bastante caso haja uma detecção precoce (de 15\% para cerca de 95\%).

Nesse contexto, estudos têm sido feito na área de aprendizado de máquina com o objetivo de facilitar a identificação precoce das lesões. Porém, um dos maiores problemas existentes nessa área é a falta de imagens anotadas disponíveis. Assim, o surgimento das redes generativas adversariais (GANs) [1], arquiteturas com o objetivo principal de gerar imagens sintéticas, se mostraram como uma possível solução para amenizar esse problema. Compostas por uma rede conhecida como geradora e outra conhecida como discriminadora, as GANs são capazes de gerar imagens inéditas, aumentando assim a base de dados. Com o intuito de avaliar as imagens geradas por essas redes, esse projeto teve como objetivo o estudo de diferentes métricas de avaliação existentes, como forma de verificar a real utilidade das pontuações fornecidas e de encontrar uma possível forma de melhorar o treinamento utilizando-as.

\section{Resultados e Discussão}

Para a obtenção dos resultados foram utilizadas três diferentes métricas: Inception Score (IS) [2], Frechét Inception Distance (FID) [3] e Sliced Wasserstein Distance (SWD) [4]. As arquiteturas de GANs foram treinadas utilizando-se três diferentes estratégias, uma delas sendo o treinamento utilizando as imagens sem as labels, e as outras duas foi utilizado o treinamento com as labels com concatenação e com multiplicação. Utilizamos um conjunto de 10015 imagens da tarefa 3 da competição ISIC 2018 e outro sendo um agrupamento de vários conjuntos de dados totalizando 26847 imagens. Coletamos também os resultados das métricas aplicadas entre o conjunto real e ele mesmo para a obtenção de um limite inferior.

Tabela 1. Resultados da métrica IS.

\begin{tabular}{|l|c|c|}
\hline \multicolumn{1}{|c|}{ Base de dados } & \multicolumn{2}{c|}{ IS } \\
\cline { 2 - 3 } & Real & Sintética \\
\hline $10015 /$ sem labels & 3.30 & 2.78 \\
\hline $10015 /$ multiplicação & 3.28 & 3.23 \\
\hline $10015 /$ concatenação & 3.28 & 3.49 \\
\hline $26847 /$ concatenação & 4.53 & 4.59 \\
\hline
\end{tabular}

Tabela 2. Resultados da métrica FID.

\begin{tabular}{|l|c|c|}
\hline \multicolumn{1}{|c|}{ Base de dados } & \multicolumn{2}{c|}{ FID } \\
\cline { 2 - 3 } & Real & Sintética \\
\hline $10015 /$ sem labels & 0 & 44.47 \\
\hline $10015 /$ multiplicação & 0 & 68.77 \\
\hline $10015 /$ concatenação & 0 & 78.99 \\
\hline $26847 /$ concatenação & 0 & 58.86 \\
\hline
\end{tabular}

Tabela 3. Resultados da métrica SWD.

\begin{tabular}{|l|c|c|}
\hline \multicolumn{1}{|c|}{ Base de dados } & \multicolumn{2}{c|}{ SWD } \\
\cline { 2 - 3 } & Real & Sintética \\
\hline $10015 /$ sem labels & 2.02 & 17.15 \\
\hline $10015 /$ multiplicação & 2.51 & 43.64 \\
\hline $10015 /$ concatenação & 2.51 & 20.61 \\
\hline $26847 /$ concatenação & 1.29 & 16.95 \\
\hline
\end{tabular}

A partir dos resultados, observamos uma grande discrepância, resultando em uma ausência de suporte base que ajudem a embasar uma conclusão com relação ao desempenho das GANs. Podemos notar também que a adição de label e o aumento no conjunto de imagens de treinamento nem sempre gera um resultado melhor nas pontuações, dificultando a identificação de fatores que contribuam para o aprendizado das redes.

\section{Conclusões}

Conseguimos observar a grande diferença nos valores e no comportamento das pontuações de cada métrica no decorrer do treinamento da GAN, evidenciando a grande dificuldade na confiabilidade e escolha de uma única métrica para a realização da avaliação das imagens. Com tantos trabalhos atualmente utilizando métricas como Inception Score para avaliar os resultados de diferentes arquiteturas de GANs, é preciso cautela, necessitando sempre de mais fatores que ajudem a fundamentar uma conclusão satisfatória.

\section{Agradecimentos}

Agradecemos ao PIBIC/CNPq, SAE/UNICAMP, FAEPEX (3125/17), FAPESP $(2017 / 16246-0)$ e Google LARA 2018.

[1] I. Goodfellow, J. Pouget-Abadie, M. Mirza, B. Xu, D. Warde-Farley, S. Ozair, A. Courville, and Y. Bengio, "Generative adversarial nets", NIPS, 2014 [2] T. Salimans, I. Goodfellow, W. Zaremba, V. Cheung, A. Radford, and X. Chen, "Improved techniques for training gans," in NIPS, 2016.

[3] M. Heusel, H. Ramsauer, T. Unterthiner, B. Nessler, and S. Hochreiter, "Gans trained by a two time-scale update rule converge to a local nash equilibrium," in NIPS, 2017

[4] T. Karras, T. Aila, S. Laine, and J. Lehtinen, "Progressive growing of gans for improved quality, stability, and variation," ICLR, 2018. 